\title{
Structural Correlations of the Lanthanide Halides and Related Compounds. Derivatives of the Anti-Nickel-Arsenide Structure
}

\author{
JOHN M. HASCHKE \\ Department of Chemistry, University of Michigan, Ann Arbor, Michigan 48109
}

Received December 29, 1975

\begin{abstract}
An examination of structural data for lanthanide halides and related compounds has shown that a substantial number of different structure types are conveniently described as layered structures derived from anti-NiAs by removal or shear of cation layers and distortion of the residual layers. The structural correlations of various $M X, M X_{1.5}, M X_{2}, M X Y, M X_{3}$, and $M X_{2} Y$ compositions ( $M=$ cation, $X$ and $Y=$ anions) are described by the presentation of a subgroup-supergroup diagram relating their space groups and by comparison of their structural projections. A close relationship between the C.SCl- and NiAs-type structures is observed. The occurrence of displacive and order-disorder phase transitions, the formation ternary derivatives by ion accommodation processes and the possible formation of intermediate halides, $M_{2} X_{2 n+1}$, by coherent intergrowth of $M X_{2}$ and $M X_{3}$ structures are discussed. The effects of radius ratio and cation coordination number on the stabilities of halide structures and on the formation of complex $M X_{2}$ layers derived from hexagonal-closest-packed metal arrays are examined.
\end{abstract}

\section{Introduction}

The lanthanide halides have an extensive halide chemistry with stoichiometries that correspond to both normal and unusual valence states. Observed compositions include $M X_{4}, M X_{3}, M X_{2}, M X_{1.5}$ phases ( $\left.I\right)(M=$ cation, $X=$ anion) as well as a large number of intermediate halides (2) which are defined by the general formula $M_{n} X_{2 n+1}$ (3). Approximately 10 years ago Thoma (4) reviewed the halides and observed that little was known about their crystal chemistry. The cubicrelated dihalide structures have since been described by Baernighausen (5), but the definition of additional structural relationships is necessary for clarification of phase transitions, coherent intergrowth, and other structurerelated phenomena of halide systems.

In a recent communication on the formation of ternary phases by anion substitution in $M^{\text {III }} X_{3}$ phases of the lanthanides and actinides (6), several similarities of the $\mathrm{UCl}_{3}$ - and $\mathrm{PuBr}_{3}$-type structures were described. The

characteristically short crystallographic axes (3.5-4.0 $\AA$ ) of these trihalide structures and their tendency toward nine-coordinate cations are observed for their substitution phases, $M X_{3-x} Y_{x}$ ( $Y=$ second anion) (6), as well as for $M X_{1.5}, M Y_{2}$, and other $M X_{3}$ halides $(1,4,7)$. These similarities suggest the existence of additional structural relationships.

The need for unifying the crystal chemistry of the halides and related materials has prompted an extensive examination of their structural data. This effort, which includes the correlation of structures and their derivation from a common highest-symmetry structure, has been aided by an elementary consideration of radius ratios and by the examination of the subgroup-supergroup relationships of the space groups.

\section{Space Group Relationships}

A subgroup diagram of the space groups derived from $P 6 / \mathrm{mmc}$ is given in Fig. 1. The subgroup relationships have been obtained 


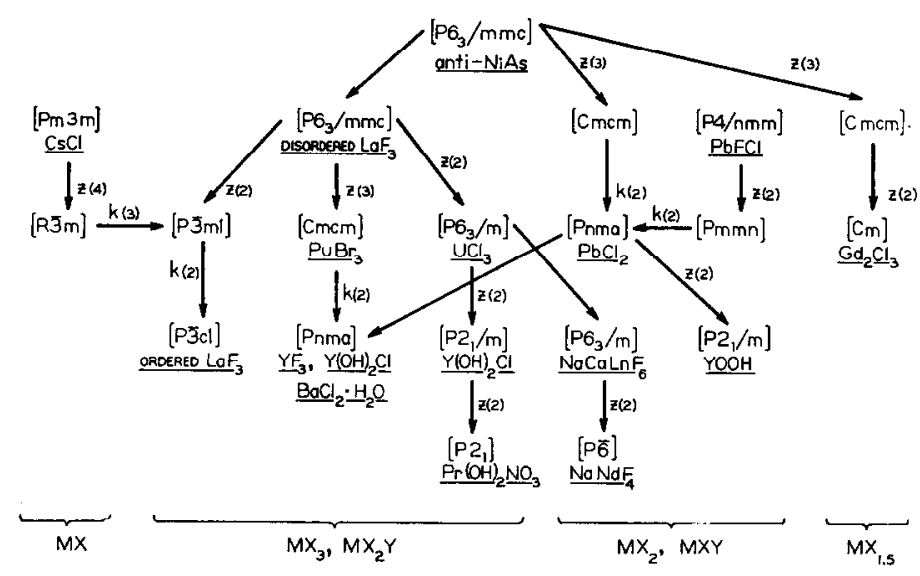

FiG. 1. Subgroup diagram of space groups derived from $P 6_{3} / m m c$.

from the tables of Neubueser and Wondratschek $(8)$ and are indicated by arrows. The symbols $z(n)$ and $k(n)$ refer, respectively, to zellengleich (same cell) and klassengleich (same class) relationships of the index $n(9)$.

The structural correlations of this report concern the various structure types shown with their respective space groups in Fig. 1. The diagram implies that the lower symmetry structures of $M X_{3}$ (plus $M X_{2} Y$ ), $M X_{2}$ (plus $M X), M X_{1.5}$, and other $M X$ phases are derivatives of the highest symmetry antiNiAs-type structure. Symmetry losses are observed for changes in structure type and for changes in both composition and structure type. Relationships indicated by unlabeled arrows involve composition changes which are not accompanied by a loss of symmetry.

The existence of subgroup-supergroup relationships between the space groups of two structures is a necessary condition for the existence of a derivative relationship, but its occurrence does not demand the presence of a close structural relationship. Figure 1 provides an overview of the structural correlations described in this report and demonstrates that the essential symmetry criteria are satisfied.

\section{Structural Relationships}

The Anti-Nickel-Arsenide Type Structure

A discussion of the structural relationships outlined in Fig. 1 is facilitated by examination of the $(11 \overline{2} 0)$ projection of the anti-NiAs structure in Fig. 2. Unique features of the NiAs-type structure have been described by

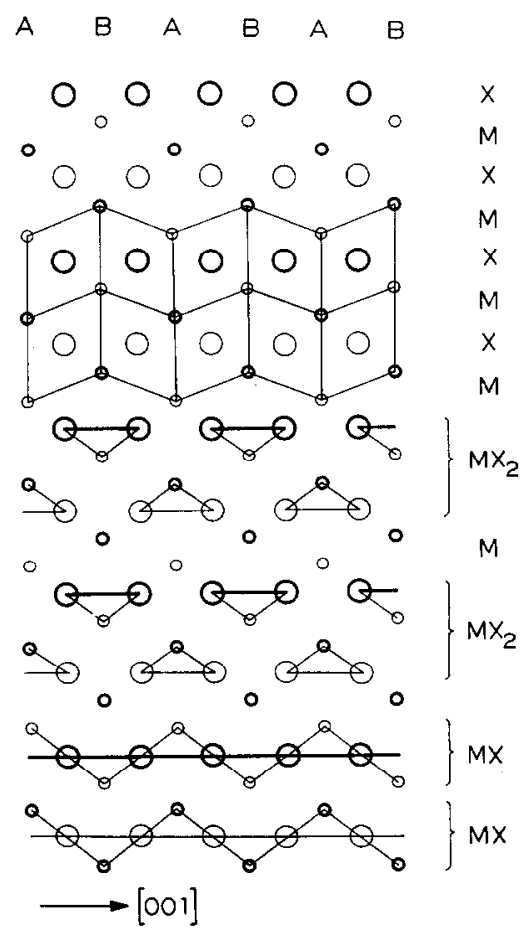

FIG. 2. The anti-NiAs-type structure projected on (1120). Large and small circles represent, respectively, anions $(X$ or $Y$ ) and cations $(M)$. Heavy and light circles are at 0 and $\frac{1}{2}$ along the projection axis, respectively.) 
Andersson and his co-workers $(10,11)$; however, the inequivalence of ionic sites in the normal and inverse structures and the use of different projection symbols in this report necessitates a brief examination of the antistructure. In Fig. 2, the projection axis is coincident with the $a$-axis of the centered orthorhombic cell of anti-NiAs $\left(a_{\text {ort }}=a_{\text {hex }}\right.$, $\left.b_{\text {ort }}=3 a_{\text {hex }}, c_{\text {ort }}=c_{\text {hex }}\right)$ and with the twofold axes of the octahedral sites in the structure. Consequently ions appear at only two levels in the structure, and the following set of identification symbols is employed in Fig. 2: Small circles represent the cation $(M)$ and large circles represent the anions ( $X$ or $Y$ ); heavy and light circles are, respectively, at fractional coordinates of 0 and $\frac{1}{2}$ along the projection axis. Except for two special cases, the ions are exclusively bilevel in all of the structure types given in Fig. 1. Therefore, the same identification symbols are used throughout this report. For consistency and ease of comparison, the origins of space groups with positional coordinates of $\frac{1}{4}$ and $\frac{3}{4}$ on the projection axis have been translated by one-quarter along the unit vector.

Examination of Fig. 2 shows that anti-NiAs may be described by two layer sequences. The usual sequence of closest-packed metal layers, $A, B, A, B, A,---$, and intervening layers of anions in octahedral sites is normal to [001]. However, the layer sequence of primary interest is parallel to [001] and may be represented by the alternation of simple metal and anion layers, $M, X, M, X, M---$, or of various complex layers such as $M X, M X, M X,---$ and $M X_{2}, M, M X_{2}, M,---$. The ions have been connected by lines which assist in the definition of the octahedral sites and in the identification of different complex layers.

\section{$\mathrm{MX}_{3}$ and $\mathrm{MX}_{2} \mathrm{Y}$ Structures}

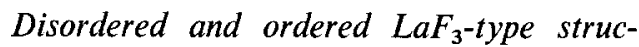
tures. Definition and clarification of the $\mathrm{LaF}_{3}$ (tysonite)-type structure has spanned a period of approximately 35 years. The original work of Oftedal (12) indicated the presence of a substructure-superstructure relationship and resulted in the assignment of a hexagonal $\left(P 6_{3} / \mathrm{mcm}, Z=6\right)$ supercell. Subsequent investigation (13) showed no evidence for the large cell, and the study of Schlyter (14) resulted in the assignment of a bimolecular hexagonal $\left(\mathrm{PG}_{3} / \mathrm{mmc}\right)$ cell. More recent examinations of high-quality synthetic crystals $(15,16)$ have verified the larger cell and shown that the space group is $P \overline{3} c 1$. Since the disordered structure is apparently observed for lanthanide and actinide trifluorides and other phases such $\mathrm{ThO}_{2} \mathrm{~F}$ and $M^{\mathrm{II}}$ (Th, $\mathrm{U} \mathrm{F}_{6}(M=\mathrm{Ca}, \mathrm{Sr}, \mathrm{Ba}$, $\mathrm{Pb})(13)$ and since it holds a key position in the correlation at trihalide structures, both the disordered and ordered structures are discussed.

Derivation of the disordered and ordered $\mathrm{LaF}_{3}$-type structures from anti-NiAs is shown in Fig. 3. In Fig. 3a, the anti-NiAs structure is represented by the layer sequence $X, M, M X_{2}$, $M, X, M X_{2}, M, X-\cdots$. A projection of the disordered $\mathrm{LaF}_{3}$ structure on (100) of the centered orthorhombic cell $\left(Z=4, a_{\text {ort }}=\right.$ $\left.a_{\text {hex }}, b_{\text {ort }}=3 a_{\text {hex }}, \quad c_{\text {ort }}=c_{\text {hex }}\right)$ is presented in Fig. 3b. Comparison of Figs. $3 a$ and $3 b$ show that shear of the anti-NiAs structure by removal of the $M$ layers from Fig. 3a produces the disordered $\mathrm{LaF}_{3}$-type structure without loss of symmetry. An alternate view of disordered $\mathrm{LaF}_{3}$ in Fig. $3 \mathrm{c}$ shows the trigonal prismatic metal coordination polyhedra which are similar to those observed in the $\mathrm{UCl}_{3}$ - and $\mathrm{PuBr}_{3}$-type structures (6). The positions of adjacent polyhedra are staggered along the projection axis such that each prism is pentacapped. The cations have an elevenfold coordination in which two $M-X$ distances (3.01 $\AA$ ) are somewhat longer than the other nine $(2.41-2.64 \AA)$.

Comparison of the ordered $\mathrm{LaF}_{3}$ structure in Fig. 3d with that of disordered $\mathrm{LaF}_{3}$ in Fig. $3 \mathrm{~b}$ shows that the superstructure arises primarily from the ordered displacement of anions within the $X$ layers. The ordered structure is projected on (100) of the centered orthorhombic cell $\left(a_{\text {ort }}=3 a_{\text {hex }}, b_{\text {ort }}=a_{\text {hex }}\right.$, $\left.c_{\text {ort }}=c_{\text {hex }}\right)$ of the trigonal $(P \overline{3} c 1)$ structure. Since this orthorhombic cell is a threefold volume multiple of the orthorhombic cell of disordered $\mathrm{LaF}_{3}$ and is derived from it by trippling the projection axis of Fig. 3b, heavy and light circles represent, respectively, ions at $0, \frac{1}{3}$, and $\frac{2}{3}$ and at $\frac{1}{6}, \frac{1}{2}$, and $\frac{5}{6}$ along the projection axis. The positional coordinates 
(a)

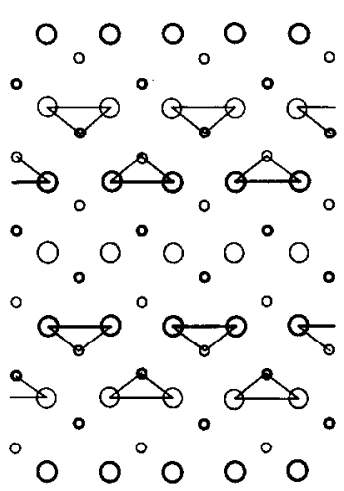

(d)

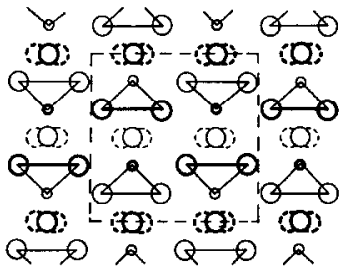

(b)

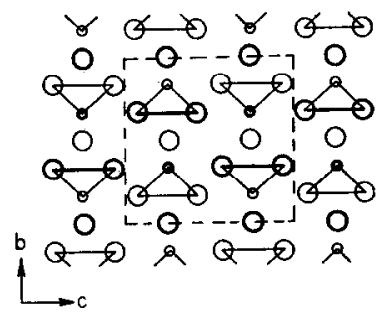

(c)

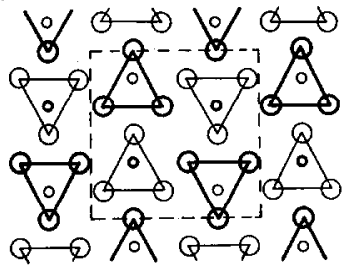

FIG. 3. The derivation of disordered and ordered $\mathrm{LaF}_{3}$-type structures from anti-NiAs. (a) The projection of anti-NiAs on (1120). (b) The structure of disordered $\mathrm{LaF}_{3}$ projected on (100) of its centered orthorhombic cell. (c) $\mathrm{A}(100)$ projection showing the trigonal prismatic coordination polyhedra in disordered $\mathrm{LaF}_{3}$. (d) The structure of ordered $\mathrm{LaF}_{3}$ projected on $(100)$ of its centered orthorhombic cell. (Because of ordering along the projection axis, heavy circles are at $0, \frac{1}{3}$, and $\frac{2}{3}$ and light circles are at $\frac{1}{6}, \frac{1}{2}$, and $\frac{5}{6}$.)

of ions in the $M X_{2}$ layers of both structures are essentially identical, but two-thirds of the anions in $X$ layers of the ordered structure have $z$ coordinates that differ by \pm 0.036 from the fixed values $\left(z= \pm \frac{1}{4}\right)$ of corresponding ions in the disordered structure. The ordered displacement of these ions $\left(0.46 \AA\right.$ in $\left.\mathrm{LaF}_{3}\right)$ is indicated by the dashed circles on both sides of the $z=\frac{1}{4}$ (solid circle) positions occupied by one-third of the ions.

$\mathrm{PuBr}_{3}$ - and $\mathrm{YF}_{3}$-type structures. The $\mathrm{PuBr}_{3}-$ and $\mathrm{YF}_{3}$-type structures are readily derived from that of disordered $\mathrm{LaF}_{3}$. Comparison of the disordered $\mathrm{LaF}_{3}$ structure in Fig. $4 \mathrm{a}$ with the projection of orthorhombic $\mathrm{PuBr}_{3}$ (17) on $(100)$ in Fig. 4b shows that the structures are very closely related. In $\mathrm{LaF}_{3}$, the arrangement of anions in the $X$ layers is planar, with $y$ coordinates of 0 or $\frac{1}{2}$; in $\mathrm{PuBr}_{3}$, their arrangement is nonplanar with $y$ coordinates of \pm 0.07 and \pm 0.43 . These displacements lower the symmetry and, in conjunction with an increase in thickness of the $M X_{2}$ layer, decrease the cation coordination number to eight.

The relationship between the structures of orthorhombic $\mathrm{YF}_{3}(18)$ and disordered $\mathrm{LaF}_{3}$ is demonstrated by comparison of Fig. 4a with the projection of $\mathrm{YF}_{\mathbf{3}}$ on (001) in Fig. 4c. Alternating layers of $M X_{2}$ and $X$ are again readily discernible. Like $\mathrm{PuBr}_{3}$, the structure has nonplanar $X$ layers. However, as the dashed lines and numbers in Fig. 4c indicate, the cations and anions are both slightly displaced $( \pm 0.04$ for $M$ and \pm 0.10 or \pm 0.14 for $X$ ) from bilevel positions ( 0 or $\frac{1}{2}$ ) along the projection axis. This distortion reduces the symmetry and decreases the cation coordination number from 11 to 9 .

The $\mathrm{UCl}_{3}$-type structure. The relationship of the $\mathrm{UCl}_{3}\left(\mathrm{Y}(\mathrm{OH})_{3}\right)$-type structure (17) to that of disordered $\mathrm{LaF}_{3}$ is shown by Figs. 5a-5c. If the trigonal prismatic coordination polyhedra of the $\mathrm{LaF}_{3}$ structure (cf. Fig. 3c) are 
(a)

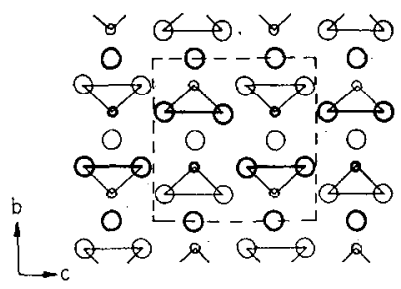

(b)

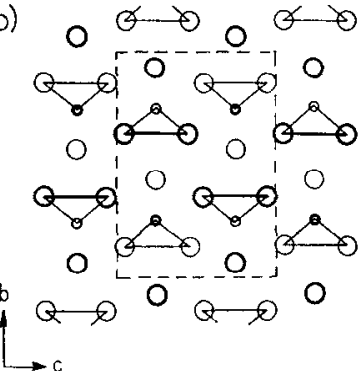

(c)

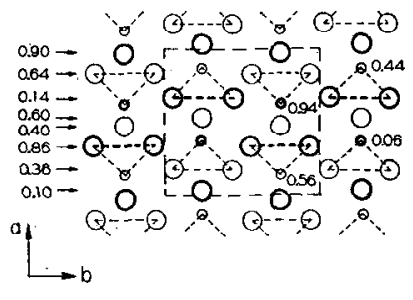

Fig. 4. The relationship of $\mathrm{PuBr}_{3}$ - and $\mathrm{YF}_{3}$-type structures to that of disordered $\mathrm{LaF}_{3}$. (a) The structure of disordered $\mathrm{LaF}_{3}$ (cf. Fig. 3b). (b) The structure of orthorhombic $\mathrm{PuBr}_{3}$ projected on (100). (c) The structure of orthorhombic $Y F_{3}$ projected on (001). (Since heavy and light circles are not at 0 and $\frac{1}{2}$, the positional coordinates for anions in layers are given at the left. Values for cations are shown within the projection.)

(a)

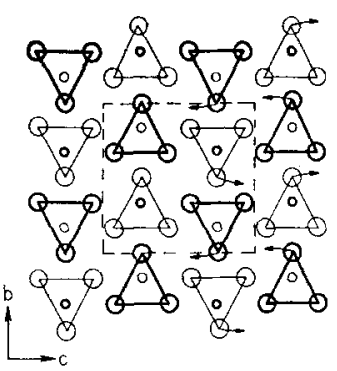

(d)

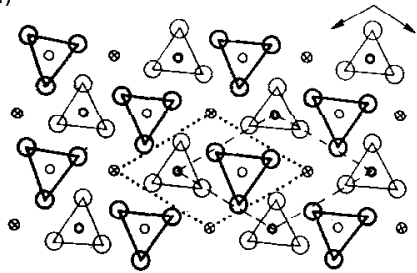

(b)

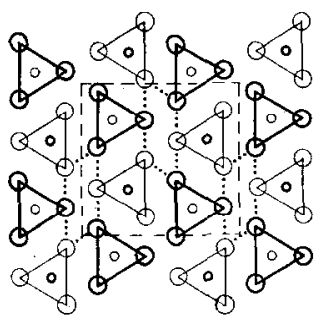

(c)

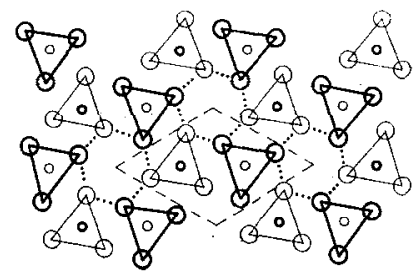

Frg. 5. The derivation of $\mathrm{UCl}_{3}$ and $\mathrm{NaNdF}_{2}$-type structures from disordered $\mathrm{LaF}_{3}$. (a) The structure of disordered $\mathrm{LaF}_{3}$ (cf. Fig. 3c). (b) An intermediate structural arrangement obtained by distortion of $\mathrm{LaF}_{3}$. (c) The structure of hexagonal $\mathrm{UCl}_{3}$ projected on (001). (c) The projection of hexagonal $\mathrm{NaNdF}_{4}$ on (001). (The positions of $\mathrm{Na}$ ions ordered at $z=0.656$ in one-half of the octahedral sites are shown by crossed circles. The projected $\mathrm{NaNdF}_{4}$ cell is indicated by dashed lines; the equivalent cell which is coincident with that of $\mathrm{UCl}_{3}$ is outlined by dots.) 
rotated slightly, as indicated by the arrows in Fig. 5a, the arrangement in Fig. $5 b$ is obtained. Several of the anions connected by dots are in positions of high electrostatic repulsion; expansion of the structure to relieve this interaction leads directly to the hexagonal $\mathrm{UCl}_{3}$-type structure which is projected on (001) in Fig. 5c. In Fig. 5b, the six coordination polyhedra connected by a given set of dots are alternately staggered by one-half unit along the projection axis, and their rectangular faces are mutually capped by adjacent prisms at each of the six interfaces within the set. During expansion, the anions involved in mutual coordination at four of the interfaces are exchanged by slippage parallel to the polyhedral faces. The resulting $\mathrm{UCl}_{3}$ structure has a symmetric ninefold cation coordination with channels of vacant octahedra normal to the projection plane. The $\mathrm{UCl}_{3}$ structure is also readily described by alternation of $M X_{2}$ and $X$ layers (6); however, the arrangement of ions within the $M X_{2}$ layers is different from that observed in the $\mathrm{LaF}_{3^{-}}, \mathrm{YF}_{3^{-}}$, and $\mathrm{PuBr}_{3^{-}}$ type structures.

Derivatives of the trihalide structures. The subgroup relationships in Fig. 1 imply that the structures of several multicomponent phases are derived from those of binary trihalides. Substitution processes (replacement of ions by other ions) and addition processes (occupancy of interstitial sites by other components) are observed methods for obtaining multicomponent derivatives of trihalide structures. The existence of alternating $\mathrm{MX}_{2}$ and $X$ layers in the trihalide structures is emphasized by the apparent facility of anion replacement in the $X$ layers. It has been demonstrated previously $(6,19)$ that total substitution of monovalent anions in the $X$ layers of $\mathrm{PuBr}_{3}-$ and $\mathrm{UCl}_{3}$-type structures leads, respectively, to the orthorhombic $\mathrm{La}(\mathrm{OH})_{2} \mathrm{NO}_{3^{-}}$and monoclinic $\mathrm{Y}(\mathrm{OH})_{2} \mathrm{Cl}-$ type structures.The lower-symmetry $\operatorname{Pr}(\mathrm{OH})_{2}-$ $\mathrm{NO}_{3}$ and twinned orthorhombic $\mathrm{Y}(\mathrm{OH})_{2} \mathrm{Cl}$ structures $(6,20)$ are closely related to that of monoclinic $\mathrm{Y}(\mathrm{OH})_{2} \mathrm{Cl}$.

Substitution of divalent anions into the disordered $\mathrm{LaF}_{3}$-type structure is apparently based on a layering sequence involving the alternation of $M X$ and $2 X$ layers. Examination of Fig. 3c shows that $M$ ions are not centered in the prismatic polyhedra, but are displaced toward the anions at $y=0$ or $\frac{1}{2}$. The $M X$ layers which are composed of these closely positioned $M$ and $X$ pairs alternate with $2 X$ layers normal to [001] of $\mathrm{LaF}_{3}$. The $P \overline{6} 2 c$ space group of $\mathrm{CeFCO}_{3}$ (bastnaesite) is a direct subgroup of $P 6_{3} / m m c(8)$, and the structure (21) is readily obtained from that of disordered $\mathrm{LaF}_{3}$ by ordered replacement of the $2 X$ layers with layers of carbonate ions.

The existence of derivative mixed-metal halides is also indicated by Fig. 1. The formation of disordered $\mathrm{LaF}_{3}$-type structures for $\mathrm{BaThF}_{6}$ and related phases (13) demonstrates isomorphous substitution processes. Structural derivatives are demonstrated by the relationships between $\mathrm{UCl}_{3}$ and $M X_{2}$ structures. Gargaranite (22), which apparently varies in composition from $\mathrm{NaCaLaF}_{6}$ to $\mathrm{Na}_{2} \mathrm{Ca}_{2} L n_{3} \mathrm{~F}_{15}(L n=$ trivalent lanthanide $)$ and $\mathrm{NaNdF}_{4}(23)$, a lower symmetry phase which is structurally related to it, are derived from $\mathrm{UCl}_{3}$ as shown by the projection of hexagonal $\mathrm{NaNdF}_{4}$ on (001) in Fig. 5d. The bimolecular $\mathrm{UCl}_{3}$-type cell of $\mathrm{NdCl}_{3}$ in Fig. $5 \mathrm{c}$ contains two vacant octahedral sites. The composition change from $M X_{3}$ to $M X_{2}$ is accomplished by the ordered placement of $M^{1}\left(\mathrm{Na}^{+}\right)$on one-fourth of the $M^{\text {III }}\left(\mathrm{Nd}^{3+}\right)$ sites and on one-half of the vacant octahedral sites. The higher symmetry $\left(P 6_{3} / m\right)$ of $\mathrm{NaCaLnF}_{6}(22)$, $\mathrm{NaYF}_{4}(24)$, and several $\mathrm{NaLnF}_{4}$ phases (23) obviously arises from the disordered accommodation of the substituted and interstitial cations.

Phase transitions of the $\mathrm{MX}_{3}$ Phases. The close similarity and facile interconversion of the $\mathrm{LaF}_{3}-, \mathrm{YF}_{3}-, \mathrm{PuBr}_{3}-$, and $\mathrm{UCl}_{3}$-type structures suggests the possibility of displacive phase transitions, which have been observed in both $\mathrm{X}$-ray diffraction and calorimetric experiments $(25-26)$. The lighter $\mathrm{YF}_{3}$-type lanthanide (Sm-Ho) trifluorides have been shown to form $\mathrm{LaF}_{3}$-type phases at high temperatures. The conversion of metastable $\mathrm{LaF}_{3}$-type $\mathrm{EuF}_{3}$ to the stable $\mathrm{YF}_{3}$-type modification at temperatures as low as $50^{\circ} \mathrm{C}$ (27) is also consistent with the close correspondence of atomic coordinates in the two structures. The $\mathrm{YF}_{3}$-type trifluorides of the 
heavier lanthanides (Er-Lu) and yttrium also exhibit thermal transitions; however, the high-temperature forms are not $\mathrm{LaF}_{3}$-type. The diffraction data for the high temperature phases (25) may be indexed on hexagonal systems which are consistent with $\mathrm{UCl}_{3}$-type structures. However, the reflection intensities are inconsistent, and the high-temperature forms of the heavier lanthanide trifluorides appear to be $\alpha-\mathrm{UO}_{3}$-type phases as proposed by Sobolev and Fedorov (28). Examination of the $\alpha-\mathrm{UO}_{3}$ structure shows that it is a distorted cubic closest packed arrangement of anions with one-third of the octahedral sites occupied by cations and that it is related to the $\mathrm{YF}_{3^{-}}$or $\mathrm{LaF}_{3}$-type structures by a simple displacive process.

\section{$\mathrm{MX}_{2}$ and $\mathrm{MXY}$ Structures}

$\mathrm{YOOH}-, \mathrm{Sr}(\mathrm{OH})_{2^{-}}, \mathrm{PbCl}_{2^{-}}$, and $\mathrm{PbFCl}$-type structures. The derivation of $M X_{2}$ structures from the anti-NiAs-type is shown in Fig. 6. The (1120) projection of anti-NiAs in Fig. 6a is represented by the layer sequence $M X_{2}, M$,

(a)

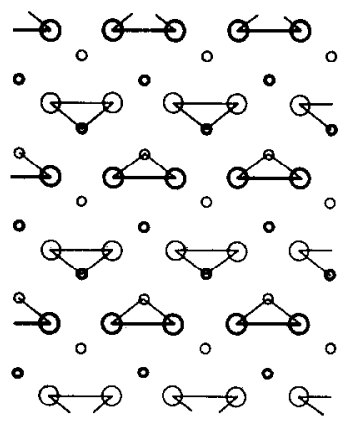

(d)

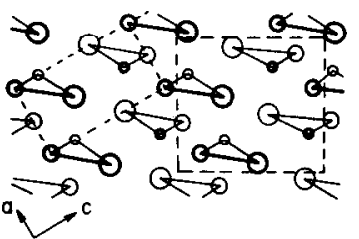

$M X_{2}, M,---$. Removal of the $M$ layers leads directly to the monoclinic YOOH-type structure (29) projected on (010) in Fig. 6b. The $M X_{2}$ layers parallel to (001) are like those observed in the $\mathrm{LaF}_{3}$ - and $\mathrm{PuBr}_{3}$-type structures; $M X_{2}$ layers like those in $\mathrm{UCl}_{3}$-type structures are parallel to (101). Although the direct relationship between the $\mathrm{UCl}_{3}$ - and YOOH-type structures is not shown in Fig. 1, it has been demonstrated in a previous report (6) that removal of $X$ layers from the $\mathrm{UCl}_{3}$ structure reduces the cation coordination from ninefold to sevenfold and that thermal decomposition of $\mathrm{UCl}_{3}$-type lanthanide trihydroxides to form YOOH-type oxide hydroxides apparently proceeds by a topotactic process.

The YOOH structure provides a convenient reference for examination of $M X_{2}$ and other $M X Y$ structures. Grueninger and Baernighausen (30) have described the orthorhombic $\mathrm{Sr}(\mathrm{OH})_{2}$-type (Pnma) structure and observed that it is closely related to that of YOOH. A slight tilting of the triangular-shaped $M X_{2}$

(b)

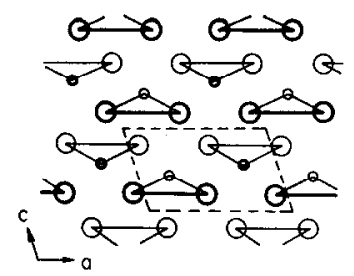

(c)

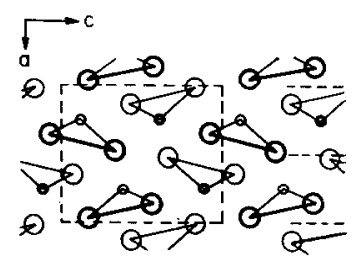

FIG. 6. The derivation of $\mathrm{YOOH}-, \mathrm{PbCl}_{2-}$, and $\mathrm{PbFCl}$-type structures from Anti-NiAs. (a) The anti-NiAs structure projected on (1120). (b) The structure of monoclinic YOOH projected on (010). (c) The Structure of orthorhombic $\mathrm{PbCl}_{2}$ projected on (010). (d) The structure of tetragonal $\mathrm{PbFCl}$ projected on (010). (The bimolecular tetragonal cell is shown at the left and a $\mathrm{PbCl}_{2}$-type cell is shown at the right.) 

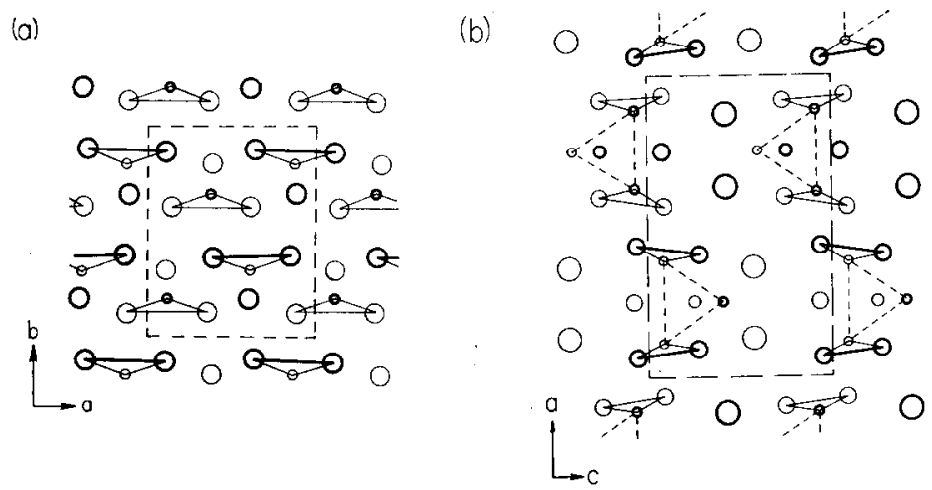

FIG. 7. Derivative and similar structures. (a) The structure of orthorhombic $\mathrm{BaCl}_{2} \cdot \mathrm{H}_{2} \mathrm{O}$ on (001). (Unattached circles indicate the oxygen positions of water molecules.) (b) The structure of orthorhombic $\mathrm{Y}_{3}(\mathrm{OH})_{5} \mathrm{OCl}$ projected on (010). (The largest circles in layers parallel to (001) represent chloride. The smallest circles in tetrahedral sites shown by dashed triangles represent oxide. Intermediate circles represent hydroxide.)

units from their orientations parallel to (100) in Fig. 6b increases the symmetry, but maintains the cation coordination number at seven. The $\mathrm{PbCl}_{2}$-type structure (31) projected on (010) in Fig. 6c also has Pnma symmetry, but the coordination number is increased to nine by a substantial tilting of the $M X_{2}$ units. Dashed lines in the right portion of Fig. 6c define the distorted $M X_{2}$ layers. Comparison of Figs. $6 \mathrm{~b}$ and $6 \mathrm{c}$ also shows that the relative positions of $M X_{2}$ layers in adjacent cells of $\mathrm{PbCl}_{2}$ are slightly displaced from those of $\mathrm{YOOH}$. The tetragonal $\mathrm{PbFCl}$-type structure (32) projected on (010) in Fig. 6d is also closely related to those of $\mathrm{YOOH}$ and $\mathrm{PbCl}_{2}$. For comparison, the projection of a tetramolecular $\mathrm{PbCl}_{2}$-type cell is indicated along with that of a bimolecular tetragonal cell. In YOOH, the oxide ions are in a slightly staggered layer about (001) (6); the hydroxides are in layers about (002). In $\mathrm{PbFCl}$, the corresponding fluoride positions are exactly confined to $(001)$ layers and the cation number is increased to eight with a ninth anion $(\mathrm{Cl})$ at $3.25 \AA$.

Derivatives of dihalide and related structures. Several of the previously described $M_{2} Y$ structures can be derived from $M X_{2}$ structures by anion accommodation processes. However, this approach is useful only for the $\mathrm{BaCl}_{2}$. $\mathrm{H}_{2} \mathrm{O}$-type structure (33) which is projected on (001) in Fig. 7a. Cation and anion positions are identified by triangular-shaped $M X_{2}$ groups which form layers of the $\mathrm{PuBr}_{3}$ (or $\mathrm{LaF}_{3}$ )-type. The lattice parameters and cation coordination geometry of $\mathrm{BaCl}_{2} \cdot \mathrm{H}_{2} \mathrm{O}$ are similar to those of $\mathrm{PuBr}_{3}$, but a careful comparison of Figs. $4 \mathrm{~b}$ and $7 \mathrm{a}$ shows that adjacent $M X_{2}$ layers are different in the two structures. Although equivalent structures are obtained by displacement of alternate layers (3), the relationship to the YOOH structure in Fig $6 \mathrm{~b}$ is simple. Addition of water molecules in the open channels between the $M X_{2}$ layers of $\mathrm{YOOH}$ leads directly to the nine-coordinate $\mathrm{BaCl}_{2} \cdot \mathrm{H}_{2} \mathrm{O}$ structure. The facile hydration of lanthanide dihalides to form crystalline monohydrates (34), which apparently exhibit variable water content (3), is entirely consistent with these structural correlations and the occurrence of a topotactic hydration reaction for $\mathrm{PbCl}_{2}$-type dihalides.

Phase transitions of $\mathrm{MX}_{2}$ and $\mathrm{MXY}$ phases. The feasibility of displacive phase transitions between $\mathrm{YOOH}-, \mathrm{Sr}(\mathrm{OH})_{2^{-}}, \mathrm{PbCl}_{2^{-}}$, and $\mathrm{PbFCl}$-type structures is clearly evident from their close structural relationships. The transition of YOOH-type phases to a highercoordinate structure is expected at high pressure. However, the potential for polymorphism of $M X_{2}$ phases is substantially more extensive because the $\mathrm{PbCl}_{2}$-type structure is also related to the $\mathrm{CaF}_{2}$-type structure by a process involving displacement of the $M X_{2}$ layers (35). Temperature- and pressure- 
induced transitions of this type are known (36) and may well include other structures which are derivatives of $\mathrm{CaF}_{2}(5)$.

\section{$\mathbf{M}_{\mathbf{2}} \mathbf{X}_{\mathbf{3}}$ Structures}

The $\mathrm{Nd}_{2} \mathrm{Cl}_{3}$-type structure. Derivation of the $\mathrm{Nd}_{2} \mathrm{Cl}_{3}$-type structure (37) from the antiNiAs-type structure is indicated in Fig. 8. The anti-NiAs structure is represented by the layer sequence $M_{2} X_{3}, M, M_{2} X_{3}, M,---$ in Fig. 8a. Removal of the $M$ layers produces the $M_{2} X_{3}$ composition in Fig. 8b. An ordered displacement of anions from their octahedral positions and into layers defined by the dashed lines is shown by the arrows in Fig. 8b. These displacements are accompanied by distortion of the residual octahedra and lead directly to the monoclinic $\mathrm{Nd}_{2} \mathrm{Cl}_{3}$-type structure projected on (010) in Fig. 8c. The strings of vacant edge-shared metal octahedra normal to the projection plane seem unusual, but the close relationship between the $\mathrm{Nd}_{2} \mathrm{Cl}_{3}$ and anti-NiAs structures demonstrates that these one-dimensional channels are fragments of the extended edge-shared octahedral arrays

(a)

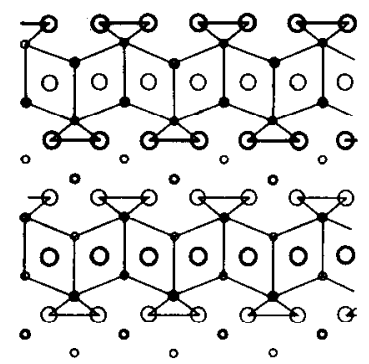

found in closest packed metals and other metalrich binary compounds (38).

Related structures. Simple derivatives of the $\mathrm{Nd}_{2} \mathrm{Cl}_{3}$ structure are not known, but a comparison of the $\mathrm{Y}_{3}(\mathrm{OH})_{5} \mathrm{OCl}_{2}$ structure (39) projected on (010) in Fig. 7b with that of $\mathrm{Nd}_{2} \mathrm{Cl}_{3}$ (Fig. 8c) shows that they are closely related. Both structures contain complex cationic layers which alternate with similar chloride-containing anion layers parallel to (001). In $\mathrm{Y}_{3}(\mathrm{OH})_{5} \mathrm{OCl}_{2}$, the cationic layers are composed of back-shared $\left[M_{2} X_{4}\right]$ columns which are intergrown with columns of $\mathrm{OM}_{4}$ tetrahedra and $\mathrm{OH}(6)$. In $\mathrm{Nd}_{2} \mathrm{Cl}_{3}$, the $\left[M_{2} X_{4}\right]$ columns are intergrown with columns of vacant metal octahedra.

\section{Other MX Structures}

Examination of the anti-NiAs structure shows that it is closely related to that of $\mathrm{CsCl}$. In Fig. $9 \mathrm{a}$, anti-NiAs is represented by the layer sequence $M X, M X, M X,-\cdots$. The relative positions of adjacent layers differ by one-half unit along the projection axis. Displacement of alternate $M X$ layers by one-

(b)

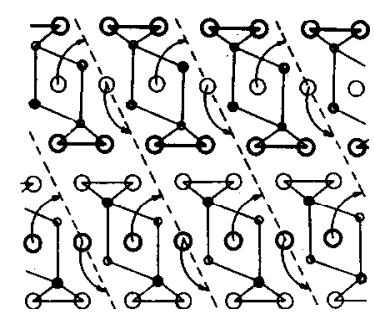

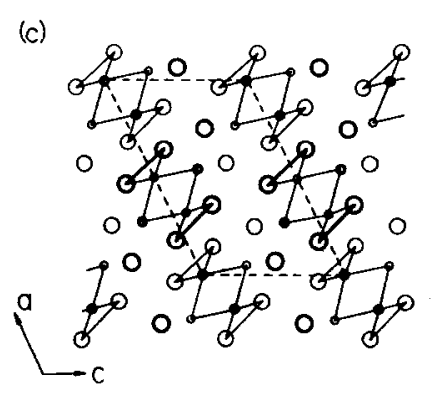

Fig. 8. The derivation of the $\mathrm{Nd}_{2} \mathrm{Cl}_{3}$-type structure from anti-NiAs. (a) The projection of anti-NiAs on (1120). (b) An intermediate structural arrangement obtained by removal of cation layers from anti-NiAs. (c) The structure of monoclinic $\mathrm{Nd}_{2} \mathrm{Cl}_{3}$ projected on (010). 
(a)
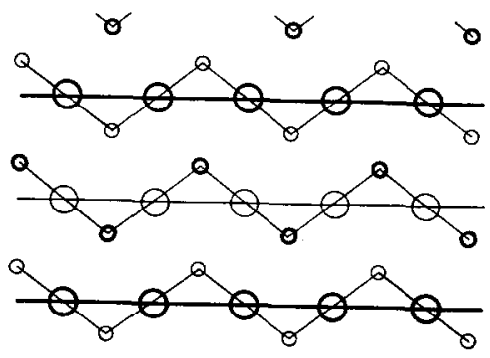

Q

Q

a

(b)

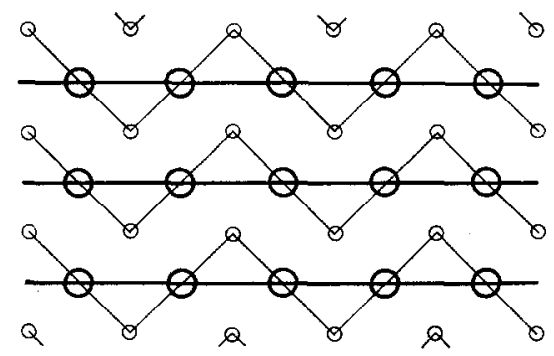

FIG. 9. The relationship between the $\mathrm{CsCl}$ - and antiNiAs-type structures. (a) The anti-NiAs structure projected on (1120); (b) The structure of cubic $\mathrm{CsCl}$ projected on (100).

half brings the anions and cations into separate levels and produces the eight-coordinate CsCl-type structure projected on (100) in Fig. 9b. The existence of this relationship apparently prccludes the crystallization of metal halides in either the normal- or antiNiAs-type structures (40). Although thermally-induced phase transitions of CsCl-type phases involve structures with sixfold coordination, the $\mathrm{NaCl}$-type polymorph is observed (41).

\section{Conclusions}

Examination of various $M X_{1.5}, M X_{2}$, and $M X_{3}$ structures has shown that they are layered structures which may be derived from the anti-NiAs structure by removal of metal layers which are parallel to [001]. A predominant feature of $M X_{2}$ and $M X_{3}$ structures is the presence of $M X_{2}$ layers which are similar to those shown for anti-NiAs in Fig. 2. An analy- sis of their origin shows that the triangular $M X_{2}$ columns normal to the projection plane are composed of an $M$ column shared by two adjacent metal octahedra and the columns of $X$ which occupy those octahedra. The $\mathrm{PuBr}_{3}-$ (or $\mathrm{LaF}_{3}$ ) type $M X_{2}$ layers, which may be anionic, neutral, or cationic, and the $\left[M_{2} X_{4}\right]$. units described previously (6) are tragments of octahedra found in the antiNiAs structure. The $M X_{2}$ layers of $\mathrm{UCl}_{3}$ and related structure types are composed of identical $\left[M_{2} X_{4}\right)$ units $(6)$; the presence of back-shared $\left[M_{2} X_{4}\right]$ units in $\mathrm{Nd}_{2} \mathrm{Cl}_{3}$ has been described previously. The characteristically short crystallographic vectors and bilevel arrangements of atomic positions in these structures are inherent features of octahedra and their fragments and of their relationship to hexagonal closest packed metal structures.

Isolation of $M X_{2}$ layers in these structures may seem arbitrary, but substantial experimental and theoretical evidence indicates that they are particularly stable arrangements. Numerous examples of $M X_{2}$ layers are found in anion substitution phases $\left(M X_{2} Y\right)$ of the lanthanides and actinides, and the phase equilibria and crystal growth habits of hydroxide anion phases are consistent with their existence (6). The relationship between $\mathrm{CaF}_{2-}$ and $\mathrm{PbCl}_{2}$-type structures (35) also depends on the existence of these layers. The apparent stability of $M X_{2}$ layers is best understood by examination of intralayer cation coordination, which is sixfold in both the $\mathrm{UCl}_{3}$ - and $\mathrm{PuBr}_{3}$ type layers. The presence of intimate sixfold coordination in essentially two dimensions permits the attainment of high coordination numbers in three dimensions. Packing of adjacent layers and, in some cases, distortion of the $M X_{2}$ layers gives cation coordination numbers of seven in $\mathrm{YOOH}$, eight in $\mathrm{PuBr}_{3}$ and $\mathrm{Y}(\mathrm{OH})_{2} \mathrm{Cl}$, nine in $\mathrm{PbCl}_{2}$ and $\mathrm{UCl}_{3}$, and eleven in $\mathrm{LaF}_{3}$. The extensive occurrence of $M X_{2}$ layers seems to reflect the stability and versatility of sixfold coordination in two dimensions.

An enhanced understanding of the crystal chemistry of lanthanide halides and related phases is obtained from the present correlations. Structural data (40), which are summarized for the lanthanide trihalides in Table $I$, 
TABLE I

Structural Data for the Lanthanide TriHalides, $M X_{3}$

\begin{tabular}{|c|c|c|c|c|c|c|}
\hline Structure type & $\mathrm{LaF}_{3}$ & $\mathrm{YF}_{3}$ & $\mathrm{UCl}_{3}$ & $\mathrm{PuBr}_{3}$ & $\mathrm{YCl}_{3}$ & $\mathrm{BiI}_{3}$ \\
\hline $\mathbf{F}$ & La-Pm & Sm-Lu & - & - & - & - \\
\hline $\mathrm{Cl}$ & - & - & La-Gd & Tb & Dy-Lu & \\
\hline $\mathrm{Br}$ & - & - & $\mathrm{La}-\mathrm{Pr}$ & Nd-Eu & - & Gd-Lu \\
\hline I & - & - & - & La-Nd & & $\mathrm{Sm}-\mathrm{Lu}^{a}$ \\
\hline$X^{-} / M^{3+}$ range $^{b}$ & $1.28-1.39$ & $1.41-1.60$ & $1.63-1.93$ & $1.96-2.17$ & $1.99-2.13$ & $2.08-2.55$ \\
\hline Cation coordinate number & 11 & 9 & 9 & 8 & 6 & 6 \\
\hline
\end{tabular}

${ }^{a} \mathrm{EuI}_{3}$ is unknown.

${ }^{b}$ Values are in part from (42) and include data for isostructural actinide compounds.

show two distinct categories, derivatives of hexagonal closest packed cation arrays and derivatives of hexagonal closest packed $\left(\mathrm{BiI}_{3}\right.$ (or $\mathrm{FeCl}_{3}-$ ) type) or cubic closest packed $\left(\mathrm{YCl}_{3}-\right.$ (or $\left.\mathrm{AlCl}_{3}-\right)$ type) anion arrays. $\mathrm{A}$ previous examination of radius ratios values has demonstrated that derivatives of closest packed anion arrays have low (sixfold) cation coordination numbers and are stable at $r X^{-}$ $r M^{3+}$ values greater than $2.0-2.2(42)$. The radius ratio boundaries for the $\mathrm{YCl}_{3^{-}}$and $\mathrm{BiI}_{3}$-type structures are rather diffuse and contrast with the well-defined limits for the derivatives of the closest packed metal arrays. A curious aspect of these data is that the closely related $\mathrm{LaF}_{3}-$ and $\mathrm{PuBr}_{3}$-type structures (cf. Fig. 4) are at opposite extremes of the radius ratio range. The expected decrease in coordination number with increase in radius ratio is observed, but the number is held constant at nine for the $\mathrm{YF}_{3}$ - and $\mathrm{UCl}_{3}$-type structures. These intermediate types apparently derive substantial stability from ninefold coordination which is achieved by structural distortion in $\mathrm{YF}_{3}$ and by the ability of ions to pack in back-shared layers (6) in $\mathrm{UCl}_{3}$.

The derivation of dihalide structures from either closest packed anion or cation arrays is also apparent. The situation is, however, less clearly defined because of the infrequent occurrence of lanthanide dihalides and because of the lack of structural information. Available data (5) show that $\mathrm{CaF}_{2-}$ and $\mathrm{PbCl}_{2}$-type structures or derivatives of $\mathrm{CaF}_{2}$ are observed at low $X^{-} / M^{2+}$ ratios. The $\mathrm{CdI}_{2}$-type struc- ture, which is derived from closest packing of anions, is observed at higher $X^{-} / M^{2+}$ ratios. These observations, the formation of antiNiAs derivatives at $M X_{1.5}$ and $M X$, and the apparent absence of relationships between $M X_{4}$ structures and closest packed metal arrays are consistent with the radius ratio trends established by the trihalides.

The utility of structural correlations in the interpretation of phase equilibria has been demonstrated by the clarification of displacive and order-disorder phase transitions and by interpretation of topotactic ion accommodation processes. However, their importance in identifying coherent intergrowth and crystallographic shear mechanisms merits comment. The localized intergrowth of $\left[M_{2} X_{4}\right]$ units with $\mathrm{OM}_{4}$ tetrahedra and $M_{6}$ octahedra has been described above, but the possibility of extended intergrowth of structures with common origins also must be recognized. Such intergrowth processes are expected if similar layers or other features exist in two phases with different compositions. They may well provide the structural mechanism for the formation of the homologous series compositions, $M_{n} X_{2 n+1}$, of the intermediate halides. Derivation of the $\mathrm{PbCl}_{2}$-type structure by shear of $\mathrm{UCl}_{3}(43)$ and the coherent intergrowth of $\mathrm{PbCl}_{2}$ - and $\mathrm{PuBr}_{3}$-type structures (3) have been described. A similar intergrowth mechanism has been proposed for the intermediate halides of the heavier lanthanides which have structures derived from closest packed anion arrays (44). 
Application of the present structural correlations in elucidating the crystal chemistry of lanthanide halides and in stimulating experimental work is anticipated. Ion accommodation processes of layered materials and structural features of the intermediate halides are recognized as important areas for examination. However, the availability of new data and the recognition of additional relationships will obviously necessitate reexamination and expansion of these correlations.

\section{References}

1. D. Brown, "Halides of the Lanthanides and Actinides," Wiley, New York (1968).

2. J. D. Corbett, Rev. Chim. Mineral. 10, 239 (1973).

3. J. M. Haschke, Inorg. Chem. 15, 298 (1976).

4. R. E. Thома, in "Progress in Science and Technology of Rare Earths," (L. Eyring, Ed.), Vol. II, pp. 90-121, Pergamon Press, New York, (1966).

5. H. Baernighausen, P. H. Beck, AND H.-W. Grueninger, "Proceedings of the Ninth Rare Earth Research Conference," National Technical Information Service, CONF-711001, Vol. 1, p. 74 (1971).

6. J. M. HaschKe, J. Solid State Chem. 14, 238 (1975).

7. T. MOELlER, in "Comprehensive Inorganic Chemistry," (J. C. Bailar, Jr., H. J. Emeléus, R. Nyholm, and A. F. Trotman-Dickenson, Eds.), Vol. 4, pp. 1-101, Pergamon Press, Oxford (1973).

8. J. Neubueser AND H. WondratscheK, "Maximal Subgroups and Minimal Supergroups of the Space Groups," private communication with H. Wondratschek, University of Karlsruhe, Karlsruhe, West Germany (1974).

9. J. Neubueser and H. WondratscheK, Krist. Tech. 1, 529 (1966).

10. S. ANDERsson ANd J. GaLy, J. Solid State Chem. 1, 576 (1970).

11. S. Andersson AND B. G. Hyde, J. Solid State Chem. 9, 92 (1974).

12. I. Oftedal, Z. Phys. Chem. 5B, 272 (1929).

13. W. H. ZACHARIASEN, Acta Crystallogr. 2, 388 (1949).

14. K. SCHLYTER, Ark. Kemi 5,73 (1953).

15. M. MansmanN, Z. Kristallogr. Kristallgeometr. Kristallphys. Kristallchem. 122, 375 (1965).

16. A. Zalkin, D. H. Templeton, AND T. E. Hopkins, Inorg. Chem. 5, 1466 (1966).

17. W. H. Zachariasen, Acta. Crystallogr. 1, 265 (1948).

18. A. ZalkIn and D. H. Templeton, J. Amer. Chem. Soc. 75, 2453 (1953).
19. J. M. Haschke, L. R. Wyles, T. A. Deline, AND D. R. PEACOR, in "Proceedings of the Eleventh Rare Earth Research Conferences," National Technical Information Service, CONF-74102-P2, p. 550 (1974).

20. M. Lundberg and A. J. Skarnulis, private communication with M. Lundberg, Arizona State University, Tempe, Ariz.

21. G. Donnay and J. D. H. Donnay, Amer. Mineral. 38, 932 (1953).

22. A. A. Voronkov, N. G. Shumyatskaya, AND Yu. A. Pyatemko, Zh. Strukt. Khim. 3, 691 (1962).

23. J. H. Burns, Inorg. Chem. 4, 881 (1965).

24. B. P. Sobolev, D. A. Mineev, ANd P. V. Pushutin, Dokl. Akad. Nauk. SSSR 150, 791 (1963).

25. R. E. Thoma AND G. D. Brunton, Inorg. Chem. 5, 1937 (1966).

26. F. H. Spedding and D. C. Henderson, J. Chem. Phys. 51, 2476 (1971); F. H. Spedding, D. J. Beaudry, D. C. Henderson, and J. Moorman, J. Chem. Phys. 60, 1978 (1974).

27. O. Greis and T. Petzel, $Z$. Anorg. Allgem. Chem. 403, 1 (1974).

28. B. P. Sobolev AND P. P. Federov, Kristallografiya 18, 624 (1973).

29. A. N. Christensen, Acta Chem. Scand. 19, 1391 (1965).

30. H. W. Grueninger and H. BaERnighausen, Z. Anorg. Allgem. Chem. 368, 62 (1969).

31. H. Baernighausen, Rev. Chim. Mineral. 10, 77 (1973)

32. W. Nieuwenkamp and J. M. Bujvoet, $Z$. Kristal$\log r .84,469$ (1932).

33. B. K. Vajnstejn and Z. G. Pinsker, Zh. Fiz. Khim. 24, 432 (1951).

34. J. M. Haschke and H. A. Eick, J. Inorg. Nucl. Chem. 32, 2153 (1970).

35. J. M. HASCHKE, High Temp. Sci. 7, 152 (1975).

36. K.-F. SEIFERT, Fortschr. Mineral. 45, 214 (1968).

37. D. A. LoKKen AND J. D. CoRbeTt, Inorg. Chem. 12, 566 (1973).

38. A. L. Bowman, Nat. Bur. Stds. (U.S.) Spec. Publ. No. 364, p. 561 (1972).

39. R. F. Klevtsova, L. P. Kozeeva, and P. V. Klevtsov, Izv. Akad. Nauk. SSSR, Neorgan. Materialy 3, 1430 (1967); Inorg. Mater. 3, 1247 (1967).

40. A. F. Wells, "Structural Inorganic Chemistry," 4th ed., Oxford Univ. Press, London (1975).

41. C. D. WEST, Z. Kristallogr. A 88, 941 (1934).

42. D. Brown, S. Fletcher, and D. G. Holah, J. Chem. Soc. A 1968, 1889 (1968).

43. B. G. Hyde, A. N. Bagshaw, S. Andersson, AND M. O'KeEfFe, Ann. Rev. Mater. Sci. 4, 43 (1974).

44. P. E. CAro, Nat. Bur. Stds. (U.S.) Spec. Publ. 364, p. 367 (1972). 